\title{
Sub-diffraction-limited resolution with squeezed light
}

Mikhail I. Kolobov ${ }^{1}$, Claude Fabre ${ }^{2}$, Pierre Scotto $^{3}$, Pere Colet $^{3}$, and Maxi San Miguel ${ }^{3}$

${ }^{1}$ Laboratoire PhLAM, Université de Lille 1, F-59655 Villeneuve d'Ascq cedex, France

${ }^{2}$ Laboratoire Kastler Brossel, Université Pierre et Marie Curie, Case 74, F-75252 Paris cedex 05, France

${ }^{3}$ Instituto Mediterráneo de Estudios Avanzados (IMEDEA, CSIC-UIB), Universitat de les Illes Balears, E-07071 Palma de Mallorca, Spain

We discuss a possibility of achieving sub-diffraction-limited resolution in coherent optical imaging using multimode squeezed light. For this purpose, we formulate a quantum theory of superresolution for object reconstruction in terms of prolate spheroidal functions. We derive the expression for ultimate resolution limit in the reconstructed object using an illumination of the full object plane by a multimode squeezed vacuum. We show that the gain in superresolution using multimode squeezed light is maximum when the Shannon number $S$ of the imaging system is smaller than unity. This situation is realized, for example, in scanning microscopy. We present some preliminary numerical simulations aiming to demonstrate the feasibility of significant superresolution for $S<1$.

Submitted to the Proceedings of the "Coherence and Quantum Optics VIII", October 16, 2001 


\section{INTRODUCTION}

A new area of quantum optics known as "quantum imaging" has emerged recently as a synthesis of the branch of quantum optics studying the spatial quantum fluctuations [1-3] with the classical optics of image formation, microscopy, resolution, et cetera. The aim of quantum imaging is to investigate the modifications introduced by the quantum-mechanical nature of light into the classical imaging. Recent publications have addressed such questions as quantum limits on measurement of small displacements [4] and quantum limits on optical resolution [5]. In particular, in these publications it was demonstrated theoretically that use of multimode squeezed light can significantly improve the resolution beyond the standard quantum limit in displacement measurements and can also beat the Rayleigh resolution limit in a diffraction-limited optical system. The theoretical prediction [4] was recently confirmed experimentally [6].

A well-known Rayleigh resolution criterion states that the resolution in an optical system is limited by diffraction on a system pupil [7]. This criterion was formulated for a simple visual observation and is based on a presumed resolving capabilities of a human eye. However, it is recognized now that modern CCD cameras allow us to achieve the performance very much exceeding that of a visual observation. For example, experimental measurements of displacements in the nanometer range has been performed to detect deflection of glass fibers [8-10], microscopic phase objects [11], movement of biological, subcellular vesicles [12], measurement of ultra-weak absorption using the mirage effect [13], or in atomic force microscopy [14]. The sensitivity of all these measurements is ultimately limited not by diffraction, but by the quantum fluctuations of the light beam used in the experiment.

The question of the role of quantum fluctuations in optical resolution is very closely related to the so-called "superresolution" techniques. Recently in a series of papers [15-18] it was shown both theoretically and experimentally that, when tightly focused beams are used for illumination with either coherent or incoherent light, the Rayleigh criterion underestimates the correct resolution limit. The authors of these papers have demonstrated that a suitably designed optical system can robustly achieve an increase in linear resolution that approaches twice the Rayleigh criterion Following these demonstrations the authors have proposed two new superresolution scanning microscopes in Refs. $[19,20]$. The superresolution in these microscopes is achieved by means of a special optical mask placed in the image plane.

Since in these demonstrations and in the proposed superresolution scanning microscopy the ultimate resolution is limited not by diffraction but by the signal-to-noise ratio in the detection scheme, we believe that further improvement in superresolution can be achieved using multimode squeezed light. As shown in numerous previous publications, such light allows one to increase the signal-to-noise ratio in different imaging schemes.

In this paper we shall present in more details as compared to Ref. [5] the quantum theory of superresolution which takes into account the quantum fluctuations of light illuminating the object and the vacuum fluctuations outside the object. This theory allows us to consider the illumination of the object with multimode squeezed light. We shall estimate qualitatively the quantum limits of resolution in the case of illumination with light in coherent state and illumination with multimode squeezed light. Finally we shall present the preliminary numerical simulations of superresolution achievable for a one-dimensional two-slit object for the Shannon number smaller that unity.

\section{CLASSICAL SUPERRESOLUTION}

For simplicity we shall consider here the case of a one-dimensional coherent optical imaging. However, our results can be easily generalized for the two-dimensional imaging and also for the case on incoherent illumination as will be shown elsewhere. The scheme of diffraction-limited coherent optical imaging is shown in Fig. 1. An object of finite size $X$ is placed in the object plane $y$. The first lens $L_{1}$ performs the Fourier transform of this object into the Fourier plane $\xi$ where a pupil of size $d$ is located. The second lens $L_{2}$ performs the inverse Fourier transform and creates an image in the image plane $x$. The finite size of the pupil introduces a finite bandwidth in the transmission of the spatial frequencies by the system, so that the image is not an exact copy of the object. 


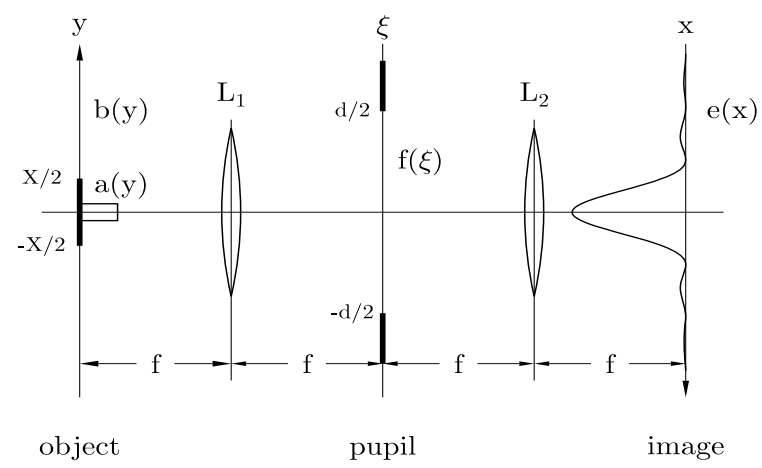

FIG. 1. Schematic of one-dimensional diffraction-limited coherent optical imaging.

"Superresolution" is an attempt to restore object details beyond the Rayleigh limit [21] or equivalently to recover the object spectrum outside the spatial-frequency band of the system. This amounts to an extrapolation of the object spatial-frequency spectrum outside the band. Such an extrapolation appears to be feasible under some assumptions, such as analyticity of the object spectrum, which holds in the case of an object of finite size $X$ (see Fig. 1). In this case the analytic continuation of the object spectrum outside the band allows in principle for unlimited resolution. However such a superresolution is extremely sensitive to the noise. The ultimate limit of superresolution is therefore determined by the quantum fluctuations of light, that is the smallest amount of noise in the optical measurement allowed by the quantum theory.

Let us introduce dimensionless variables $s=2 x / X, s^{\prime}=2 y / X$, and the space-bandwidth product $c=\frac{\pi d X}{2 \lambda f}$. In terms of these variables the transformation $L$ of the classical object amplitude $a\left(s^{\prime}\right)$ into the image amplitude $e(s)$ reads:

$$
\begin{aligned}
e(s) & \equiv(L a)(s) \\
& =\int_{-1}^{1} \frac{\sin \left[c\left(s-s^{\prime}\right)\right]}{\pi\left(s-s^{\prime}\right)} a\left(s^{\prime}\right) d s^{\prime}, \quad-\infty<s<\infty .
\end{aligned}
$$

The problem of reconstruction of the object $a\left(s^{\prime}\right)$ from a detected image $e(s)$ in the absence of noise is equivalent to inversion of the integral operator $L$. The operator $L^{\star}$ adjoint to $L$ is given by [15]

$$
\left(L^{\star} f\right)\left(s^{\prime}\right)=\int_{-\infty}^{\infty} \frac{\sin \left[c\left(s^{\prime}-s\right)\right]}{\pi\left(s^{\prime}-s\right)} f(s) d s, \quad\left|s^{\prime}\right| \leq 1 .
$$

The product $A=L^{\star} L$ is the self-adjoint operator,

$$
(A f)(s)=\int_{-1}^{1} \frac{\sin \left[c\left(s-s^{\prime}\right)\right]}{\pi\left(s-s^{\prime}\right)} f\left(s^{\prime}\right) d s^{\prime}, \quad|s|,\left|s^{\prime}\right| \leq 1,
$$

studied by Slepian and Pollak [22]. The orthonormal system of eigenfunctions of $A$ is given by

$$
\varphi_{k}\left(s^{\prime}\right)=\frac{1}{\sqrt{\lambda_{k}}} \psi_{k}\left(s^{\prime}\right), \quad\left|s^{\prime}\right| \leq 1,
$$

where $\psi_{k}(s)$ are the linear prolate spheroidal functions [22,23], and $\lambda_{k}$ are the corresponding eigenvalues. The functions $\varphi_{k}(s)$ form a basis in $L^{2}(-1,1)$ and may be considered as "elements of information" of the input object. The eigenvalues $\lambda_{k}$ are an infinite set of real, positive numbers obeying $1 \geq \lambda_{0}>\lambda_{1}>\ldots>0$. For small $k$ the $\lambda_{k}$ fall off slowly with $k$ until the index reaches the critical value, $k=S$, called the Shannon number,

$$
S=\frac{2 c}{\pi}=\frac{d X}{\lambda f}
$$

beyond which the $\lambda_{k}$ rapidly approach zero.

Using the fundamental properties of the linear prolate spheroidal wave functions, 


$$
\begin{gathered}
\int_{-1}^{1} \frac{\sin \left[c\left(s-s^{\prime}\right)\right]}{\pi\left(s-s^{\prime}\right)} \psi_{k}\left(s^{\prime}\right) d s^{\prime}=\lambda_{k} \psi_{k}(s), \\
\int_{-\infty}^{\infty} \frac{\sin \left[c\left(s-s^{\prime}\right)\right]}{\pi\left(s-s^{\prime}\right)} \psi_{k}\left(s^{\prime}\right) d s^{\prime}=\psi_{k}(s),
\end{gathered}
$$

we obtain

$$
L \varphi_{k}=\sqrt{\lambda_{k}} \psi_{k}, \quad L^{\star} \psi_{k}=\sqrt{\lambda_{k}} \varphi_{k} .
$$

Note that the functions $\psi_{k}(s)$ are defined on the real axis $-\infty<s<\infty$, and the functions $\varphi_{k}\left(s^{\prime}\right)$ on the interval $-1 \leq s^{\prime} \leq 1$. Expanding the object amplitude over the functions $\varphi_{k}\left(s^{\prime}\right)$ and the image amplitude over $\psi_{k}(s)$, we can easily find the relation between the expansion coefficients of the object and the image. Indeed, since the functions $\varphi_{k}\left(s^{\prime}\right)$ form a complete orthonormal set in $[-1,1]$ we can write the object amplitude as

$$
a\left(s^{\prime}\right)=\sum_{k=0}^{\infty} a_{k} \varphi_{k}\left(s^{\prime}\right), \quad\left|s^{\prime}\right| \leq 1,
$$

with the coefficients $a_{k}$ given by

$$
a_{k}=\int_{-1}^{1} a\left(s^{\prime}\right) \varphi_{k}\left(s^{\prime}\right) d s^{\prime}
$$

A similar expansion can be written for the image amplitude in terms of functions $\psi_{k}(s)$

$$
e(s)=\sum_{k=0}^{\infty} e_{k} \psi_{k}(s), \quad-\infty<s<\infty .
$$

Substituting these expansions into Eq. (1) and using first of Eqs. (8) we obtain the following relation between $a_{k}$ and $e_{k}$ :

$$
e_{k}=\sqrt{\lambda_{k}} a_{k} .
$$

Let us denote by $\tilde{a}_{k}$ the expansion coefficients of the object reconstructed from the measured image $e(s)$. In the absence of noise these coefficients are obtained by dividing the image coefficients $e_{k}$ by $\sqrt{\lambda_{k}}$. Thus, for a noise-free image the object reconstruction can be performed exactly, $\tilde{a}_{k}=a_{k}$, i. e. without resolution limit. The ultimate accuracy in the determination of $\tilde{a}_{k}$ will therefore be determined by quantum fluctuations in the measurement of $e_{k}$.

\section{QUANTUM THEORY OF SUPERRESOLUTION}

In the quantum theory the object amplitude $a\left(s^{\prime}\right)$ and the image amplitude $e(s)$ become operators obeying the standard commutation relations (see, for example [1]). We can use Eqs. (9),(11) now treating the expansion coefficients $a_{k}$ and $e_{k}$ as photon annihilation operators. The operators $a_{k}$ in the object plane obey the following commutation relations:

$$
\left[a_{k}, a_{l}^{\dagger}\right]=\delta_{k l}, \quad\left[a_{k}, a_{l}\right]=0 .
$$

The same commutation relations must be satisfied by the image coefficients $e_{k}$. However, Eq. (12) does not preserve the commutation relations (13). The reason for this is that classical imaging equation (1) takes into account only nonzero field amplitude in the region $\left|s^{\prime}\right| \leq 1$ of the object plane. The rest of this plane $\left|s^{\prime}\right|>1$ is ignored because there the classical field amplitude is zero. In the quantum theory this region must be taken into account to guarantee the conservation of the commutation relations.

To include the region $\left|s^{\prime}\right|>1$ in the object plane into the theory we introduce another set of functions:

$$
\chi_{k}\left(s^{\prime}\right)=\frac{1}{\sqrt{1-\lambda_{k}}} \psi_{k}\left(s^{\prime}\right), \quad\left|s^{\prime}\right|>1
$$


Using the following properties of the prolate spheroidal wave functions,

$$
\begin{aligned}
& \int_{-\infty}^{\infty} \psi_{k}(s) \psi_{l}(s) d s=\delta_{k l} \\
& \int_{-1}^{1} \psi_{k}(s) \psi_{l}(s) d s=\lambda_{k} \delta_{k l}
\end{aligned}
$$

it is easy to show that functions $\chi_{k}\left(s^{\prime}\right)$ form a complete orthonormal set in the region $\left|s^{\prime}\right|>1$. Therefore, we can use them as a basis for expansion of the field outside the object.

Now the operator of the total field in the object plane, $-\infty<s^{\prime}<\infty$, can be written as

$$
a\left(s^{\prime}\right)=\sum_{k=0}^{\infty} a_{k} \varphi_{k}\left(s^{\prime}\right)+\sum_{k=0}^{\infty} b_{k} \chi_{k}\left(s^{\prime}\right)
$$

where the operators $b_{k}$ satisfy the commutation relations similar to Eq. (13). Substituting the expansion (17) into Eq. (1) we obtain the following relation between the coefficients in the object and the image plane,

$$
e_{k}=\sqrt{\lambda_{k}} a_{k}+\sqrt{1-\lambda_{k}} b_{k}
$$

It is easy to verify that this transformation preserves the commutation relations of the operators, $\left[a_{k}, a_{l}^{\dagger}\right]=\left[b_{k}, b_{l}^{\dagger}\right]=$ $\left[e_{k}, e_{l}^{\dagger}\right]=\delta_{k l}$.

Equation (18) is completely equivalent to the transformation performed by a beam splitter. Indeed, if we consider the operators $a_{k}$ and $b_{k}$ as the photon annihilation operators in the modes defined by prolate spheroidal waves incoming to the beam splitter with the amplitude transmission coefficient $\sqrt{\lambda_{k}}$ and the reflection coefficient $\sqrt{1-\lambda_{k}}$, then $e_{k}$ is the photon annihilation operator in the $k$ th mode of the transmitted wave.

From Fig. 1 one may think that the vacuum fluctuations coming from the region $|\xi|>d / 2$ in the Fourier plane outside the pupil should be also taken into account. Indeed, when treating the field in the Fourier plane as an operator we must include the contribution from this region into the resulting field in the image plane. However, the advantage of expansion (11) is that the field from this region does not contribute to the expansion coefficients $e_{k}$ of the image because it is orthogonal to the prolate spheroidal wave functions. This property was pointed out by Bertero and Pike in [15] for the out-of-band classical noise and remains valid in the quantum theory as we proceed to prove now.

For this proof we shall use the following property of the linear prolate spheroidal functions,

$$
\int_{-1}^{1} \psi_{k}(s) e^{i \omega s} d s=i^{k}\left(\frac{2 \pi \lambda_{k}}{c}\right)^{1 / 2} \psi_{k}(\omega / c)
$$

which can be found, for example, in Ref. [23]. Taking Fourier transform of both sides of Eq. (19),

$$
\int_{-\infty}^{\infty} e^{-i \omega s^{\prime}} \int_{-1}^{1} \psi_{k}(s) e^{i \omega s} d s d \omega=2 \pi \int_{-1}^{1} \psi_{k}(s) \delta\left(s^{\prime}-s\right) d s=i^{k}\left(\frac{2 \pi \lambda_{k}}{c}\right)^{1 / 2} \int_{-\infty}^{\infty} \psi_{k}(\omega / c) e^{-i \omega s^{\prime}} d \omega
$$

and using the property of the $\delta$-function, we arrive at the following result,

$$
\int_{-\infty}^{\infty} \psi_{k}(s) e^{i \omega s} d s=\left\{\begin{array}{cc}
i^{k}\left(\frac{2 \pi}{\lambda_{k} c}\right)^{1 / 2} \psi_{k}(\omega / c), & \text { if }|\omega| \leq c \\
0, & \text { if }|\omega|>c
\end{array}\right.
$$

From this equation it follows that $\psi_{k}(s)$ is a bandlimited function with vanishing Fourier spectrum outside the region of frequencies $|\omega| \leq c$.

Let us now consider the field operator $f(\omega)$ on the left side of the pupil plane of Fig. 1. This field is related to the total field operator in the object plane through the transformation performed by the lens L1,

$$
f(\omega)=\frac{X}{2} \frac{1}{\sqrt{\lambda f}} \int_{-\infty}^{\infty}[a(s) p(s)+b(s)(1-p(s))] e^{i \omega s} d s
$$

with $\omega=2 \xi c / d$ being the dimensionless spatial frequency. Here we have used the characteristic function $p(s)$ for the interval $[-1,1]$ equal to unity inside this interval and zero outside it, which allows us to write the total field operator 
in the object plane in compact form. The field operator $e(s)$ in the image plane can be represented as the sum of two contributions coming from the area inside the pupil, $|\omega| \leq c$, and outside it, $|\omega|>c$,

$$
e(s)=\frac{d}{2 c} \frac{1}{\sqrt{\lambda f}}\left[\int_{|\omega| \leq c} f(\omega) e^{-i \omega s} d \omega+\int_{|\omega|>c} \tilde{f}(\omega) e^{-i \omega s} d \omega\right]
$$

where $f(\omega)$ is just given by Eq. (22), whereas $\tilde{f}(\omega)$ is the operator associated with the unknown field present in the area of the pupil plane located outside the pupil.

From the first sight it seems that both contributions in Eq. (23) into $e(s)$ have to be taken into account. Let us however evaluate the contributions of these two areas into the coefficient $e_{k}$ form the Eq. (11):

$$
e_{k}=\int_{-\infty}^{\infty} e(s) \psi_{k}(s) d s=\int_{|\omega| \leq c} f(\omega) \int_{-\infty}^{\infty} \psi_{k}(s) e^{-i \omega s} d s d \omega+\int_{|\omega|>c} \tilde{f}(\omega) \int_{-\infty}^{\infty} \psi_{k}(s) e^{-i \omega s} d s d \omega .
$$

As follows from Eq. (21), the second term in the sum in Eq. (24) vanishes. Thus, there is no contribution from the quantum-mechanical field operator $\tilde{f}(\omega)$ outside the pupil into all the coefficients $e_{k}$ and therefore into the field $e(s)$ in the image plane.

We will assume that we use a homodyne detection technique of the image that allows us to register any of the quadrature components of the field $e(s)$,

$$
e(s)=e_{1}(s)+i e_{2}(s)
$$

Using Eq. (18) we can express the variances of the expansion coefficients $e_{1 k}$ and $e_{2 k}$ of these quadrature components through the variances of coefficients $a_{1 k}, a_{2 k}$ and $b_{1 k}, b_{2 k}$ in the object plane,

$$
\left\langle\left(\Delta e_{\mu k}\right)^{2}\right\rangle=\lambda_{k}\left\langle\left(\Delta a_{\mu k}\right)^{2}\right\rangle+\left(1-\lambda_{k}\right)\left\langle\left(\Delta b_{\mu k}\right)^{2}\right\rangle,
$$

with $\mu=1,2$ for corresponding quadratures. From Eq. (12) the fluctuations of the expansion coefficients of the reconstructed object are obtained as follows,

$$
\left\langle\left(\Delta \tilde{a}_{\mu k}\right)^{2}\right\rangle=\frac{\left\langle\left(\Delta e_{\mu k}\right)^{2}\right\rangle}{\lambda_{k}}
$$

Let us assume that the field in the object plane is in a multimode coherent state at any point $\left|s^{\prime}\right| \leq 1$. Since there is no light outside the region $[-1,1]$ this corresponds to the vacuum state of all operators $b_{k}$. In this case the variances of the coefficients $a_{\mu k}$ and $b_{\mu k}$ in the object plane are equal to $\left\langle\left(\Delta a_{\mu k}\right)^{2}\right\rangle=\left\langle\left(\Delta b_{\mu k}\right)^{2}\right\rangle=\frac{1}{4}$, and the variances of the coefficients for the reconstructed object read

$$
\left\langle\left(\Delta \tilde{a}_{\mu k}\right)^{2}\right\rangle=\frac{1}{4 \lambda_{k}}
$$

As the eigenvalues $\lambda_{k}$ become rapidly very small for $k>S$, the corresponding variance will become very large which will forbid a precise determination of the coefficient $\tilde{a}_{\mu k}$. Therefore, formula (28) sets the standard quantum limit in reconstruction techniques used in superresolution.

Since imaging equation in the form (18) is equivalent to the transformation of two fields by a beam splitter it gives us an idea on how to reduce the fluctuations in the reconstructed object below the standard quantum limit. It is well known that such an improvement can be achieved by illuminating the open port of the beam splitter by a squeezed vacuum with properly chosen squeezed quadrature. Since in our case the role of such an open port is played by the region $\left|s^{\prime}\right|>1$ outside the object, we expect that superresolution beyond the standard quantum limit can be achieved by illuminating this region by a multimode squeezed vacuum with a light spot much larger than the size of the object. Such a light can be produced by a traveling-wave optical parametric amplifier (OPA) or a degenerate optical parametric oscillator (OPO) below threshold in a confocal cavity [1].

We may expect that an even better result for the object reconstruction can be obtained when not only the area outside the object but the object itself is illuminated by multimode squeezed light with nonzero mean amplitude. However, to use the advantage of such illumination we have to make sure that squeezing in the incoming light is not destroyed by absorption in the object. Therefore, squeezed light illumination should be advantageous for pure phase or for weakly absorbing objects.

In practice, one can use a single source of multimode squeezed vacuum with a large transverse area and mix it with a coherent light wave in the central part illuminating the object using a weakly reflecting small size mirror. This 
coherent light wave is needed for producing a nonzero field component in the image plane and plays the role similar to the local oscillator in the homodyne detection technique. The quantum fluctuations of this coherent light wave can be eliminated by using the balanced homodyne detection of two images with two CCD cameras.

To evaluate the fluctuations of the reconstructed object in this case we assume for the sake of simplicity that squeezing has infinitely large spatial bandwidth, i. e. is the same for all coefficients $a_{k}$ and $b_{k},\left\langle\left(\Delta a_{\mu k}\right)^{2}\right\rangle=\left\langle\left(\Delta b_{\mu k}\right)^{2}\right\rangle=\frac{1}{4} e^{ \pm 2 r}$, where the "-" sign corresponds to the squeezed quadrature $\mu=1$, and the "+" sign to the stretched one, $\mu=2$, and $r$ is the squeezing parameter. This gives

$$
\left\langle\left(\Delta \tilde{a}_{\mu k}\right)^{2}\right\rangle=\frac{1}{4 \lambda_{k}} e^{ \pm 2 r}
$$

It follows from this equation that the variance of the reconstruction coefficients $\tilde{a}_{1 k}$ in the squeezed quadrature component $\tilde{a}_{1}\left(s^{\prime}\right)$ can be significantly reduced below the standard quantum limit for large squeezing, $r \gg 1$. For $r=0$ we recover the standard quantum limit of Eq. (28).

To estimate the resolution length $D$ (the smallest object detail reconstructed from the image) from Eq. (28) and its improvement obtained using Eq. (29) we will use the arguments similar to Ref. [15]. For definiteness we shall consider an amplitude object, i. e. an object recorded in the amplitude quadrature component of the field, $\left\langle a^{\dagger}\left(s^{\prime}\right) a\left(s^{\prime}\right)\right\rangle=\left\langle a_{1}\left(s^{\prime}\right) a_{1}\left(s^{\prime}\right)\right\rangle$. Let us define the signal $S$ and the noise $B$ related to the reconstructed object as

$$
S=\int_{-1}^{1}\left\langle\tilde{a}_{1}\left(s^{\prime}\right) \tilde{a}_{1}\left(s^{\prime}\right)\right\rangle d s^{\prime}, \quad B=\int_{-1}^{1}\left\langle\left(\Delta \tilde{a}_{1}\left(s^{\prime}\right)\right)^{2}\right\rangle d s^{\prime} .
$$

These quantities can be evaluated as $S=\langle N\rangle$, where $\langle N\rangle$ is the total number of photons in the object, and

$$
B \simeq \frac{e^{-2 r}}{4} \sum_{k=0}^{Q} \frac{1}{\lambda_{k}} \simeq \frac{e^{-2 r}}{4} \frac{1}{\lambda_{Q}}
$$

Here $Q$ is the index of the highest eigenfunction in the reconstructed object. In the second equality we have used the fact that $\lambda_{Q}$ is the smallest eigenvalue and estimated the sum as $1 / \lambda_{Q}$. Setting the signal-to-noise ratio equal to unity we can find the smallest eigenvalue $\lambda_{Q}$ that can be recovered from the image measurement,

$$
\lambda_{Q} \simeq \frac{e^{-2 r}}{4\langle N\rangle}
$$

Using the tables of eigenvalues $\lambda_{k}$ [23] or calculating them numerically we can evaluate the index $Q$ of the highest eigenfunction $\varphi_{Q}(s)$ with a precisely known coefficient in the expansion of the reconstructed object. Knowing that $\varphi_{Q}(s)$ has exactly $Q$ zeros on the interval $[-1,1]$ we can estimate the resolution length $D$ as

$$
D \simeq X /(Q+1)=R\left(\frac{S}{Q+1}\right)
$$

where $R=X / S=\lambda f / d$ is the Rayleigh resolution length. From this equation we can interpret the number $Q+1 \equiv M$ as an effective number of degrees of freedom in the object which, as follows from Eq. (32), is a function of the total photon number $\langle N\rangle$ and the squeezing parameter $r$. The case of superresolution corresponds to $D<R$ or, equivalently, $M>S$. For classical noise the dependence of $M$ on the classical signal-to-noise ratio was studied in [15,21] with an important conclusion that significant superresolution can be achieved for small values of the Shannon number $S$. Since our Eq. (32) is an extension to the quantum domain of analogous equation studied in Refs. [15,21] we conclude that a similar result holds true for quantum fluctuations.

A scanning optical microscope is operated in a small Shannon number configuration [24]. It is therefore a good candidate for a practical application of our analysis. We will consider it in more details in a forthcoming paper together with the influence of the finite pixel size in the image plane and the finite coherence area of squeezed light in the object plane.

\section{NUMERICAL SIMULATIONS OF SUPERRESOLUTION}

We now turn to a numerical study of the superresolution problem with the aim, on one side, to demonstrate practically the possibility of resolution beyond the Rayleigh limit and, on the other side, to describe quantitatively some of the qualitative results derived in the previous section. 
Our ultimate goal is to investigate numerically the role of quantum fluctuations in the superresolution and the possibility of improving the superresolution using multimode squeezed light. However, before attacking the problem with quantum fluctuations in the detected object, we would like to verify that we are not limited by numerical errors in achieving superresolution without quantum fluctuations. These numerical errors in our simulations may be considered as technical noise in a real experiment and at this stage we would like to make sure that our "technical noise" is much smaller than the quantum noise that we intend to "measure".

To calculate the prolate spheroidal functions $\psi_{k}(s)$ and the corresponding eigenvalues $\lambda_{k}$ we have decided to solve numerically the Fredholm integral equation (6). We followed the method proposed in [25]. Evaluating the integral in Eq. (6) by means of a Gauss-Legendre quadrature [26], we obtain the following discrete form of this equation,

$$
\sum_{j=1}^{N} w_{j} \frac{\sin \left[c\left(s_{i}-s_{j}\right)\right]}{\pi\left(s_{i}-s_{j}\right)} \psi_{k}\left(s_{j}\right)=\lambda_{k} \psi_{k}\left(s_{i}\right),
$$

which involves the Gauss-Legendre weights $w_{j}$ and the zeros of the Legendre polynomials $s_{j}, j=1, \ldots, N$ as specified by the Gaussian quadrature algorithm [26]. Those were evaluated using standard algorithms [27]. Eq. (34) can be directly solved with standard numerical methods for matrix diagonalization which give access to the eigenvalues $\lambda_{k}$ and the corresponding eigenvectors, i. e. the values of the prolate functions $\psi_{k}\left(s_{j}\right)$ at the $N$ Gauss-Legendre abscissas $-1 \leq s_{j} \leq 1$. To compute the value of the prolate functions $\psi_{k}(s)$ at any arbitrary point $s$ and in particular outside the interval $[-1,1]$, we use again Eq. (6), valid for any value of $s$, in its discretized form:

$$
\psi_{k}(s)=\frac{1}{\lambda_{k}} \sum_{j=1}^{N} w_{j} \frac{\sin \left[c\left(s-s_{j}\right]\right.}{\pi\left(s-s_{j}\right)} \psi_{k}\left(s_{j}\right),
$$

which only involves on the right-hand-side the quantities previously determined from the diagonalization procedure. In Fig. 2 we illustrate two prolate spheroidal function calculated using this procedure for the typical value of the Shannon number $S=0.6$ which will be used throughout our numerical simulations.
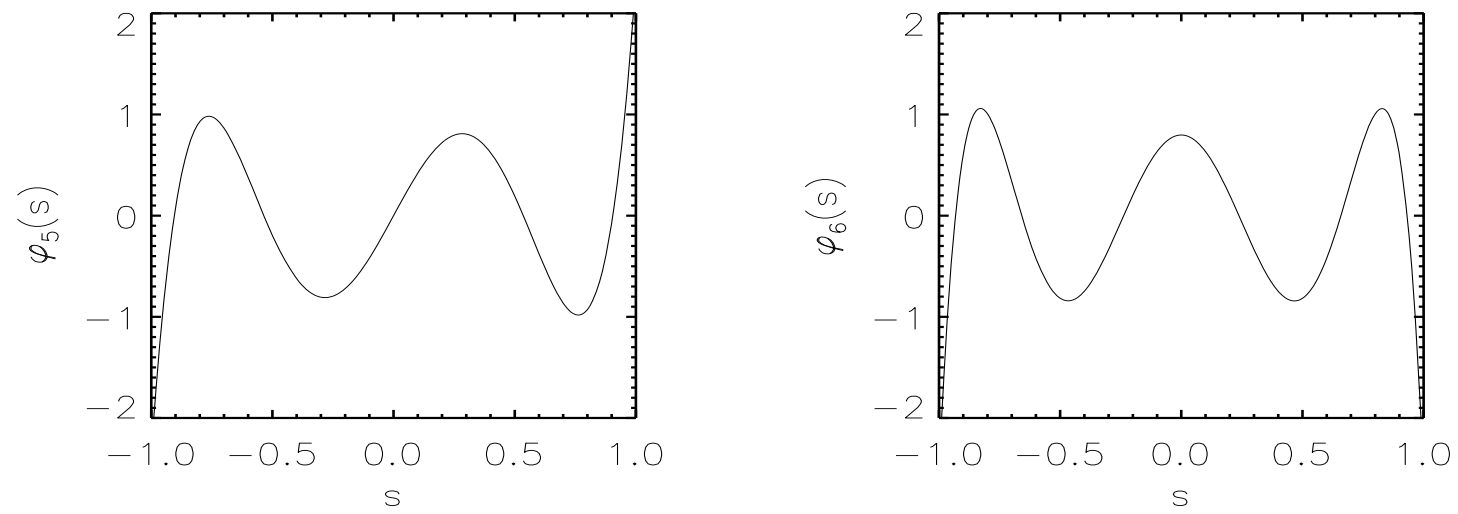

FIG. 2. Eigenfunctions $\varphi_{5}(s)$ and $\varphi_{6}(s)$ given by Eq. (4) for the Shannon number $S=0.6$.

One would expect to access the higher and higher eigenvalues $\lambda_{k}$ and the corresponding eigenfunction $\psi_{k}(s)$ by increasing the number of points $N$ used for the Gauss-Legendre quadrature. Practically, however, only a finite number of eigenvalues and eigenvectors can be calculated with accuracy. This is essentially due to the fact that eigenvalues $\lambda_{k}$ decrease extremely rapidly as a function of $k$ for $k>S$. In a numerical calculation involving sums and subtractions, like typically a diagonalization routine, this is a fundamental problem. Roughly speaking, this will prevent from precise calculation of any eigenvalue smaller than the numerical precision of the machine, which is typically of the order of $10^{-16}$, and a fortiori of the corresponding eigenvector. This numerical noise represents a strong limitation: for a typical value $S=0.6$ of the Shannon number and using double precision computation we found that only seven first eigenvalues and eigenfunctions could be computed with reliable accuracy.

Going back to the reconstruction problem, the fact that we only have a finite number of functions $\psi_{k}(s)$ at disposition is equivalent to the truncation of the exact summation formula (9). As a consequence, the reconstruction method will be imperfect. But even truncated, this procedure represents a substantial improvement with respect to the Rayleigh resolution limit, as will be illustrated in the following. For this purpose, we considered an object consisting of two 
slits of finite width $\epsilon$, separated by a given distance $b$. For a Shannon number $S=0.6$, the intensity distribution in the image plane, plotted in Fig. 3, clearly shows that the Rayleigh resolution criterium does not allow us to resolve the slits: for this Shannon number, the width of the diffraction spot associated with each slit is much larger than the distance between the slits.

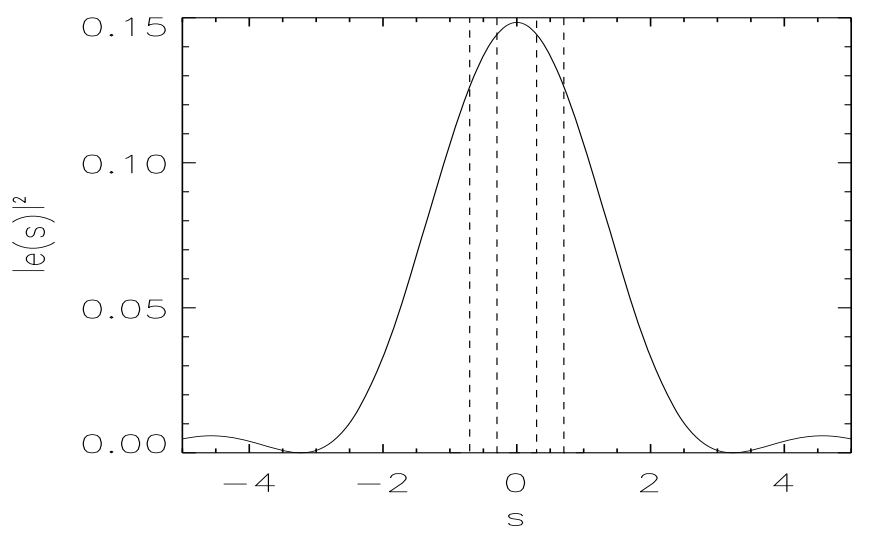

FIG. 3. Intensity distribution in the image plane for a two-slit object. For comparison, the position of the two slits in the object plane has been included as a dotted line. The distance between the slits is $b=1$, and their width $\epsilon=0.4$.

However, the result of the truncated reconstruction procedure

$$
a_{\mathrm{r}}(s)=\sum_{k=0}^{6} a_{k} \varphi_{k}(s),
$$

which is represented in Fig. 4, together with the exact object for comparison, allows for good resolution of the two slits. The difference between the object $a(s)$ and the reconstructed object $a_{\mathrm{r}}(s)$ is of course due to the fact that only seven first terms in the exact expansion (9) are taken into account.

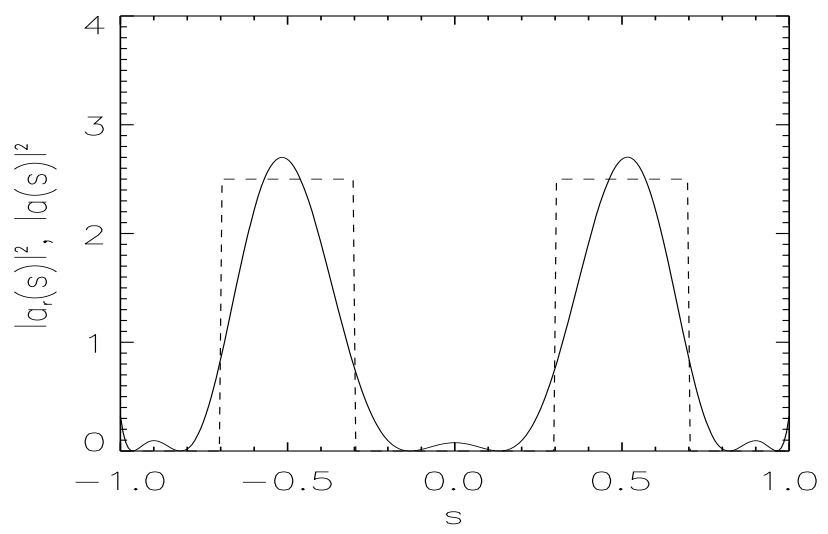

FIG. 4. Comparison of the object intensity distribution $|a(s)|^{2}$ (dotted line) with the intensity distribution $\left|a_{\mathrm{r}}(s)\right|^{2}$ (solid line). The parameters are the same as in Fig. 3.

This first result allows us to conclude that the reconstruction method with prolate spheroidal functions gives a significant improvement with respect to the Rayleigh criterium. In order to quantify this improvement, we can define the equivalent of the Rayleigh length associated with this method as the smallest distance between two-point objects which can be resolved. Here, we choose a somewhat different approach, which leads to quantitatively equivalent results: we first decreased the width of each slits, keeping constant the distance between them. For reasonably broad slits, the width of the reconstructed object follows the width of the object itself, as shown in Fig. 4. However, it turns out that in the limit of small widths, the width of the reconstructed object finally does not depend any more on the width of the object but saturates at a finite value. This limiting case is plotted in Fig. 5. In analogy to the 
original definition of the Rayleigh length, the width of each peak in the reconstructed object can be identified with the minimal length that can be resolved in $a_{\mathrm{r}}(s)$. Roughly, it corresponds to the variation scale of the highest prolate function $\psi_{6}(s)$ used in the truncated sum.

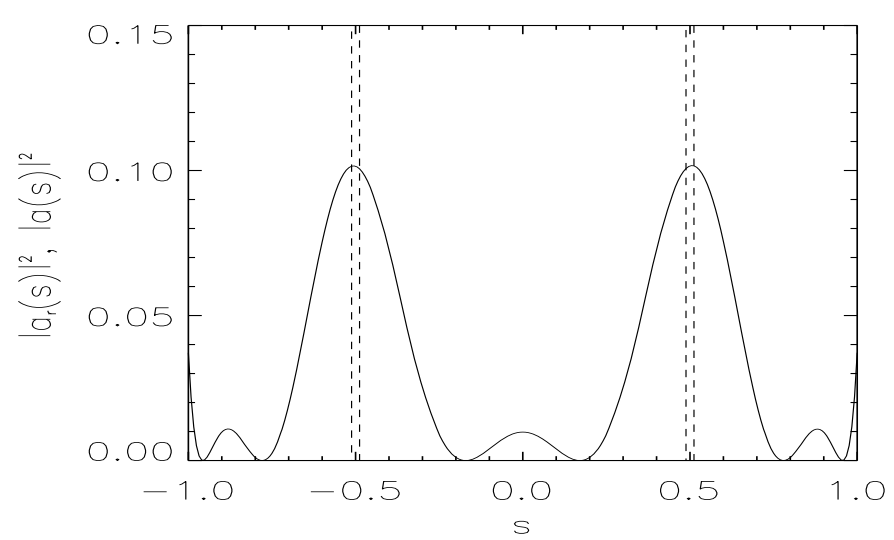

FIG. 5. Reconstructed object in the limit of very thin slits. $\epsilon=0.01$. The distance between each maximum of the intensity distribution and the first zero represents the new resolution length allowed by the reconstruction procedure.

The improvement of resolution with respect to the classical Rayleigh limit can be quantified by simply comparing this ultimate resolution length with the Rayleigh length, which can be read out from Fig. 3 as the distance between the maximum and the first zero of the intensity distribution. In the case of $S=0.6$ considered here, we find a substantial improvement by a factor of more than 10 .

It should be noted that such an improvement is possible only for Shannon numbers $S$ of the order or less that unity. Our simulations with larger Shannon numbers from $S=5$ to $S=7$ confirm that much lesser superresolution can be achieved in these cases. For example, for $S=5$ we have found a superresolution factor of 2.2 .

In conclusion, our preliminary numerical simulations of the two-point object reconstruction using prolate spheroidal functions for Shannon numbers smaller than unity demonstrate a possibility of superresolution of one order of magnitude over the Rayleigh limit. This is an encouraging result for further development of our project which aims to take into account numerically the quantum fluctuations of the illuminating light wave. We hope that simulations with multimode squeezed light as illuminating source will allow us to demonstrate an appreciable superresolution for $S<1$.

\section{ACKNOWLEDGMENTS}

This work was supported by the Network QUANTIM (IST-2000-26019) of the European Union. The Laboratoire Kastler Brossel of the Ecole Normale Supérieure and the Université Pierre et Marie Curie is associated with the Centre National de la Recherche Scientifique.

[1] M. I. Kolobov, Rev. Mod. Phys. 71, 1539 (1999).

[2] L. A. Lugiato, M. Brambilla, and A. Gatti, in Advances in Atomic, Molecular, and Optical Physics, Vol. 40, edited by B. Bederson and H. Walther (Academic, Boston, 1999), p. 229.

[3] A. Gatti, E. Brambilla, M. I. Kolobov, L. A. Lugiato, J. Opt. B: Quant. Semiclass. Opt. 2, 196 (2000).

[4] C. Fabre, J. B. Fouet, and A. Maître, Opt. Lett. 25, 76 (2000).

[5] M. I. Kolobov and C. Fabre, Phys. Rev. Lett. 85, 3789, (2000).

[6] N. Treps, U. Andersen, B. Buchler, P. K. Lam, A. Maître, H. Bachor, C. Fabre, "Crossing the standard quantum limit for high-sensitivity measurements in optical images using non-classical light", submitted to Nature (2001).

[7] J. Rayleigh, in Collected Optics Papers of Lord Rayleigh, part A, (Opt. Soc. Am., Washington,1994), p. 117.

[8] A. Flock and D. Strelioff, Nature, 310, 397 (1984). 
[9] J. J. Art, A. C. Craftford, and R. Fettiplace, J. Physiol. (London)371, 18P (1986).

[10] S. Kamimura, Appl. Opt. 26, 3425 (1987).

[11] W. Denk and W. W. Webb, Appl. Opt. 29, 2382 (1990).

[12] J. Jelles, B. J. Schnapp, and M. P. Scheetz, Nature, 331, 450 (1988).

[13] D. Fournier, A. Boccara, N. Amer, and R. Gerlach, Appl. Phys. Lett. 37, 519 (1980).

[14] C. Putman, B. De Grooth, N. Van Hulst, and J. Greve, J. Appl. Phys. 72, 6 (1992).

[15] M. Bertero, and E. R. Pike, Opt. Acta 29, 727 (1982).

[16] M. Bertero, P. Boccacci, and E. R. Pike, Opt. Acta 29, 1599 (1982).

[17] M. Bertero, P. Brianzi, P. Parker, and E. R. Pike, Opt. Acta 31, 181 (1984).

[18] M. Bertero, C. De Mol, E. R. Pike, and J. G. Walker Opt. Acta 31, 923 (1984).

[19] J. G. Walker, E. R. Pike, and M. Bertero, British patent89/13129 (6 July 1989).

[20] R. E. Davies, Ph. D. dissertation (Department of Physics, King's College, University of London,1990).

[21] M. Bertero, and C. De Mol, in Progress in Optics Vol. XXXVI, edited by E. Wolf (North-Holland, Amsterdam, 1996), p. 129.

[22] D. Slepian and H. O. Pollak, Bell System Tech. J. 40, 43 (1961);

[23] B. R. Frieden, in Progress in Optics Vol. IX, edited by E. Wolf (North-Holland, Amsterdam, 1971), p. 311.

[24] T. Wilson and C. J. R. Sheppard, Theory and Practice of Scanning Optical Microscopy (Academic Press, London, 1984).

[25] W. P. Latham and M. Tilton, Appl. Opt. 26, 2658 (1987).

[26] B. Carnahan, H. A. Luther and J. O. Wilkes, Applied Numerical Methods (Wiley, New York, 1969), p. 100.

[27] W. H. Press, B. P. Flannery, S. A. Teukolsky and W. T. Vetterling, Numerical Recipes (Fortran Version) (Cambridge University Press, Cambridge, 1989), p. 125. 